\title{
FAILURE MECHANISM RESEARCHES OF MATERIAL SURFACE AND INTERFACE IN MICRO-SCRATCH TEST
}

Yueguang Wei, Manhong Zhao, and Shan Tang

LNM, Institute of Mechanics, Chinese Academy of Sciences, Beijing 100080, P.R. China

\begin{abstract}
In the present research, the adhesion properties and failure mechanisms for a ductile thin film on a silicon substrate $(\mathrm{Ni} / \mathrm{Si})$ are studied experimentally, and are simulated theoretically. In the experimental research, the relations of the horizontal driving force, vertical displacement and the frictional coefficients with horizontal displacement are measured. Furthermore, the variation of the total energy release rate and the frictional coefficient between contact surfaces are measured through obtaining a frictional effect law. The law displays that the frictional influences on the energy release rate of the total system weakly depend on the thin film thickness. This conclusion leads to that the frictional effect can be eliminated in the toughness ratio relation approximately. So that one can directly obtain the interfacial adhesion toughness from measurements in the micro-scratching test. In addition, the micro-scratching process for the ductile thin film/brittle substrate systems is simulated using the double cohesive zone model. Prediction results of the energy release rate are obtained, and are compared with the experimental results obtained in the present research.
\end{abstract}

\section{INTRODUCTION}

The micro-scratch test is an important experimental method for determining the material surface property and the adhesion properties of thin film or coating layer on substrates. The method is often applied to the failure assessments in the large scale or super-large scale electronic circuits and the MEMS device. However, in most cases, due to the complexities of the material system and circumstances, it is often difficult to obtain the effective measurements using the experimental approach. For example, the adhesion strength and toughness for ductile film/brittle substrate system are usually very difficult to be measured due to the plastic dissipation of the thin film and the frictional dissipation.

On the researches of the material surface properties and thin film adhesion work and strength along the substrate interface, many experimental researches based on the scratch methods have been carried out in past decade [1-7]. However, very few researches on the frictional effects were explored. Obviously, the frictional effect is very important in the scratch test. On the other hand, theoretical researches (or mechanics analyses) connected with the scratch experiments are also very few in the past [1]. This is probably because that any theoretical study must deal with on the complicated failure geometry of the scratch test. Clearly, a three-dimensional elastic-plastic deformation problem must be solved, and a robust theoretical model for describing the scratch failure behavior is needed. Most theoretical researches have been based on the simple geometry of the scratch failure strap and the simple mechanics 
equilibrium to simulate the scratch failure behavior [1-7]. However, it is difficult to use a simple model to describe the strong influence of plastic deformation on the micro-scratch behavior. It is well known that plastic deformation has a strong shielding effect on the interface cracking [8].

In the present research, the adhesion properties and failure mechanisms for a ductile thin film system on a silicon substrate $(\mathrm{Ni} / \mathrm{Si})$ are studied in detail experimentally and are simulated theoretically. In the experimental research, the relations of the horizontal driving force, vertical displacement and frictional coefficients with horizontal displacement are measured. Furthermore, the variation of the total energy release rate and the frictional coefficient between contact surfaces are measured through obtaining a frictional effect law. In addition, the micro-scratching process for the ductile thin film/brittle substrate systems is simulated using the double cohesive zone model [9]. Prediction results of the energy release rate will be found, and will be compared with the experimental results.

\section{DESCRIPTION OF MICRO-SCRATCH TEST}

From failure characteristics for ductile film scratching, the scratch test process can be described by figure 1(a). This process comprises two stages. One stage is a normal scratch before thin film delamination occurs along interface. With the indenter moving forward and downward with scratch depth increase, especially when indenter tip is near the interface, a region of thin film or coating layer near the indenter tip will be delaminated from the interface. Thereby, the scratch process is transferred to another stage. The failure character changes from the indenter grooving growth to the delaminated film strap formation and growth (or post-scratch process). For simplifying the analysis, the problem is divided into two sub-problems [8]. One problem is "plate bend" under elastic-plastic large deformation for the delaminated thin film part BCD, see figure 1(a). This sub-problem is easily to be solved in theoretical analysis. Another problem is a three-dimensional delaminating problem for a part of thin film BA and jointed substrate.

\section{EXPERIMENTAL RESULTS OF TWO KINDS OF MATERIAL SYSTEMS}

The micro-scratch experiment for a $\mathrm{Ni} / \mathrm{Si}$ system are carried out. The specimen preparation and the experimental procedures are described as follows. The specimen is prepared in the Micro-Electronic Institute of Peking University. The films are deposited on the surface of single-crystal silicon along (100) surface using the test machine Research II, Sputtered Film Co., US, under the conditions of $4.6 \mathrm{mTorr}$, Ar15SCCM, and 1000W. The specimens comprise the several different thicknesses of films. The micro-scratch experiments are made on the Nanoindenter II Instrument. During the scratch experiments, different vertical loading within the fixed time interval (50s) are exerted on the specimens for different film thicknesses.

Figure 2 (a) (c) show the experimental results of the total horizontal driving force and the vertical displacement variations with the horizontal displacement for three different thin film thicknesses. From figure 2(a) (c), during the process of scratching, the total horizontal force increases as either the vertical or horizontal displacement increases. When the vertical displacement value approaches the film thickness, i.e., the indenter tip approaches the interface, 
the film is delaminated along the interface abruptly (from figure 2 (d)). This phenomenon is similar to that of the scratch experiment for the $\mathrm{Pt} / \mathrm{NiO}$ system in [6]. Thereafter, the interface crack is created and advances along the interface for a while, and goes down within the silicon (substrate) due to the test instrument programming automatically. The horizontal driving force increases abruptly when the indenter tip approaches the interface during scratching, thereafter, a stable value the the horizontal force in reached with the crack advancing along the interface, soon the horizontal force increases with the indenter tip moving forward deviating inside the substrate.

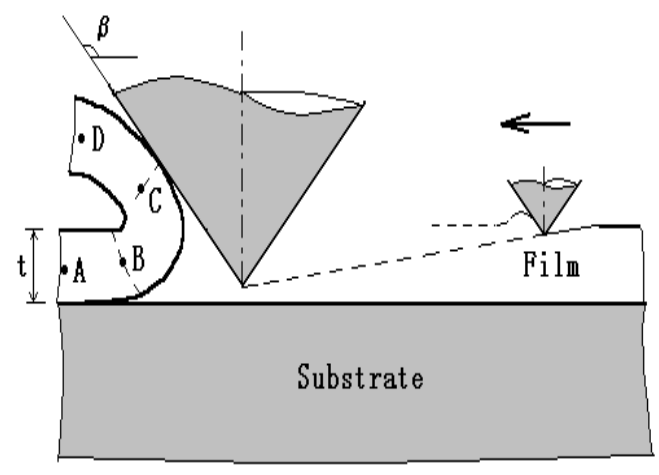

(a)

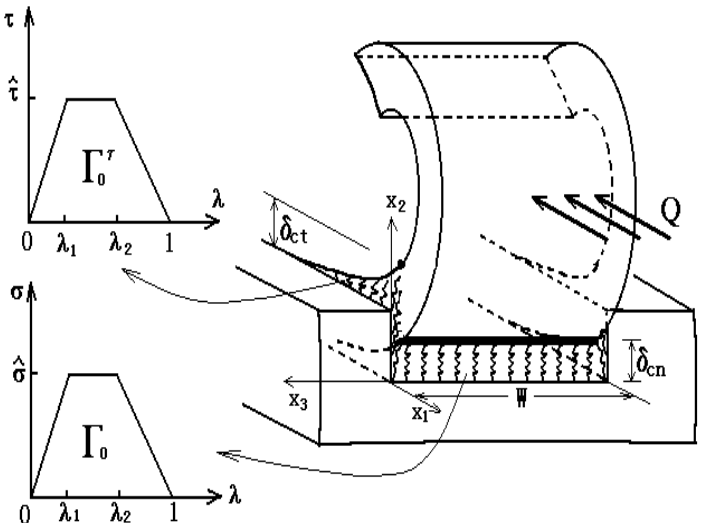

(b)

Figure 1. The sketch of the micro-scratch test and the simplified model.

Figure 3 show the variation of the frictional coefficient with the horizontal displacement during the scratching for two thin film thickness cases. From figure 3, the frictional coefficient variation changes weakly with the film thickness. So that one can neglect the friction effect in calculating the energy release rate through letting the following equality

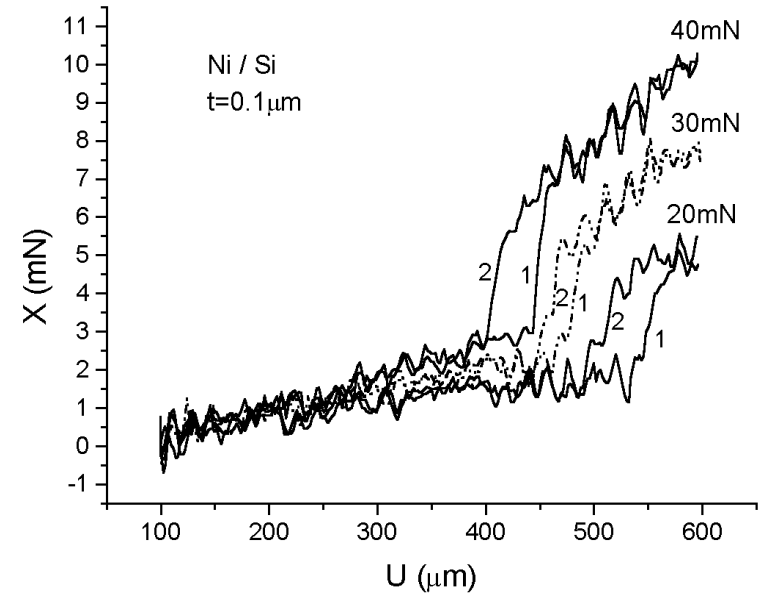

(a)

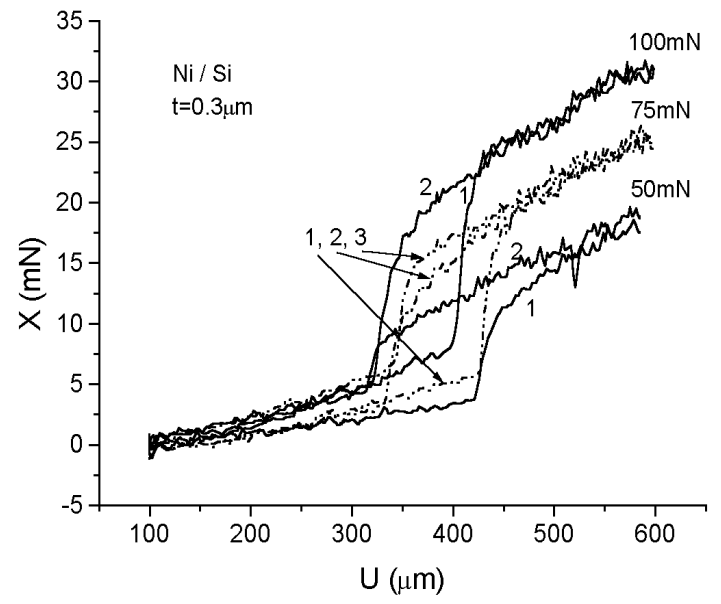

(b) 


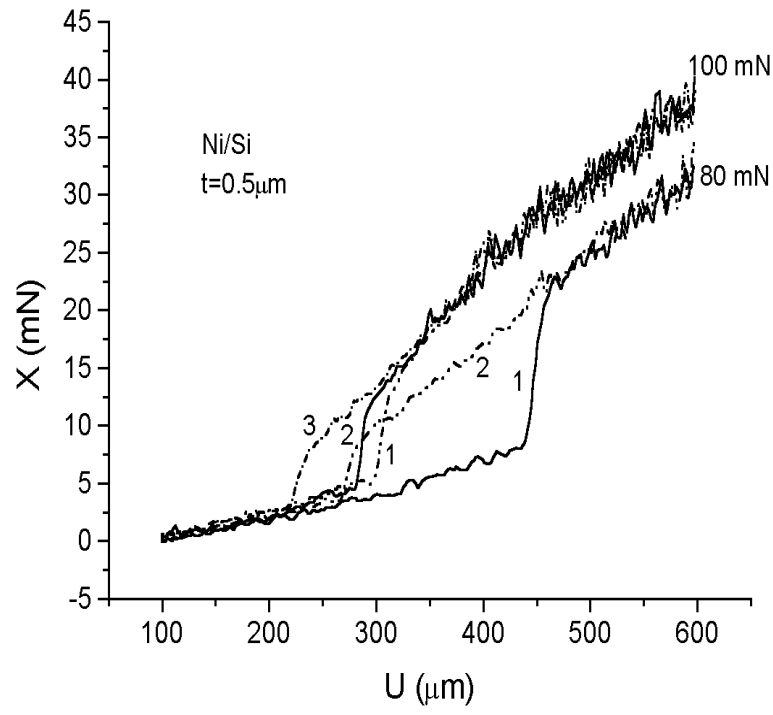

(c)

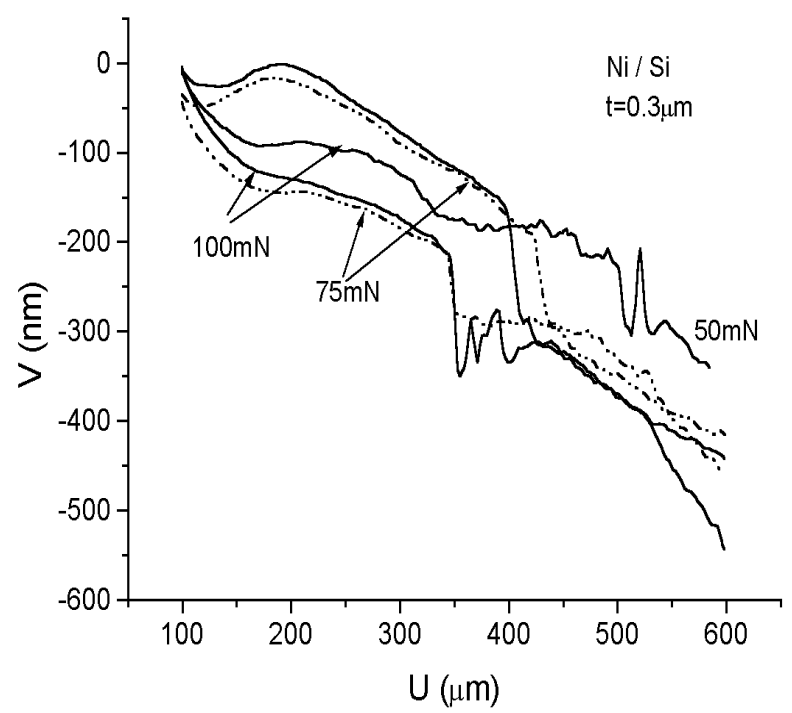

(d)

Figure 2. The relations of the horizontal driving forces ((a) (c)) and the vertical displacement (d) with horizontal displacement. Number in the figure corresponds to the different specimen.

$$
\frac{Q}{\Gamma_{0}}=\frac{X_{c r}}{X_{0}}
$$

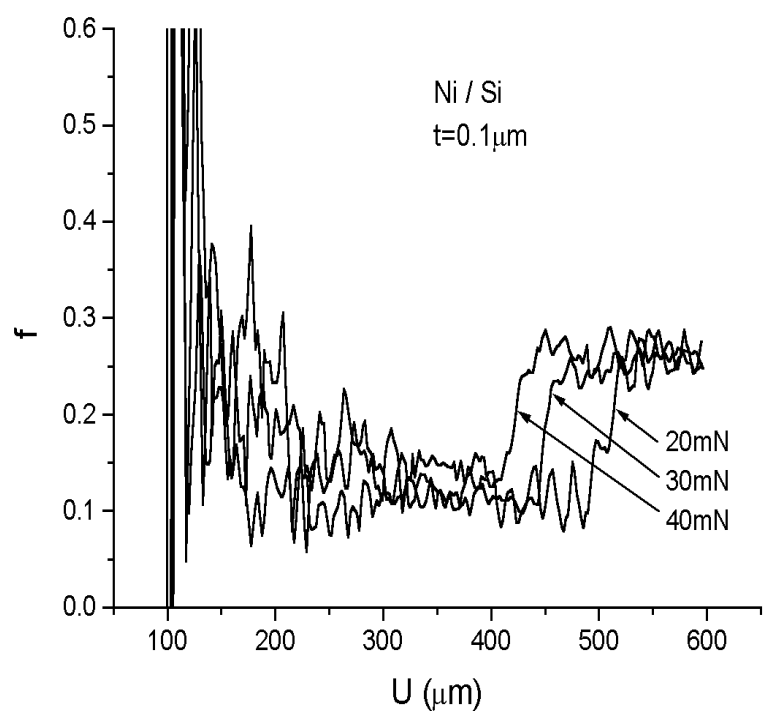

(a)

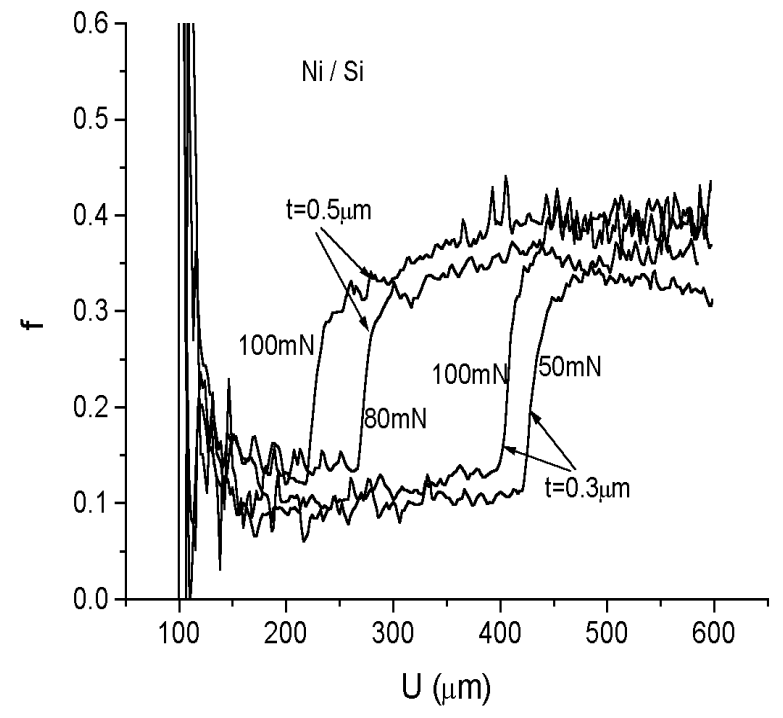

(b)

Figure 3. The relations of the frictional coefficients with horizontal displacement for three film thickness cases. 
where $Q / \Gamma_{0}$ is the normalized energy release rate, $X_{c r} / X_{0}$ is the horizontal driving force ratio. From (1), one can calculate the normalized the energy release rate by deleting the thin film thickness effect.

\section{THEORETICAL ANALYSIS OF THE ENERGY RELEASE RATE}

Double cohesive zone model (figure 1(b)) and a three dimensional elastic-plastic finite element analysis are adopted to predicted the energy release rate relation [9]

$$
\frac{Q}{\Gamma_{0}}=1+\left(\frac{2 t}{W}\right)\left(\frac{\hat{\tau}}{\hat{\sigma}}\right)+\frac{\Gamma_{P}}{\Gamma_{0}}=f\left(\frac{E}{\sigma_{Y}}, \frac{\hat{\sigma}}{\sigma_{Y}}, \frac{\hat{\tau}}{\sigma_{Y}}, \frac{t}{R_{0}}, \frac{t}{W}, N, v, \beta\right)
$$

In formula (2) a reference length parameter $R_{0}$ has been introduced, whose definition is $R_{0}=E \Gamma_{0} /\left[3 \pi\left(1-v^{2}\right) \sigma_{Y}^{2}\right]$, characterizing the plastic zone size in vertical direction in small scale yielding. The other parameters are defined in the figure 1(b).

Figure 4 show the relations of the normalized energy release rate predicted (or called horizontal driving force) $Q / \Gamma_{0}$ to the normalized thin film thickness and for several indenter tip angles. From figure 4 and from our analysis, all the parameters have considerably influences on the total energy release rate (horizontal driving force). In figure 4, the present experimental results for material system $\mathrm{Ni} / \mathrm{Si}$ obtained from figure 2 (a) (c) by means of using (1) and the experimental results from the literature [10] are also shown.

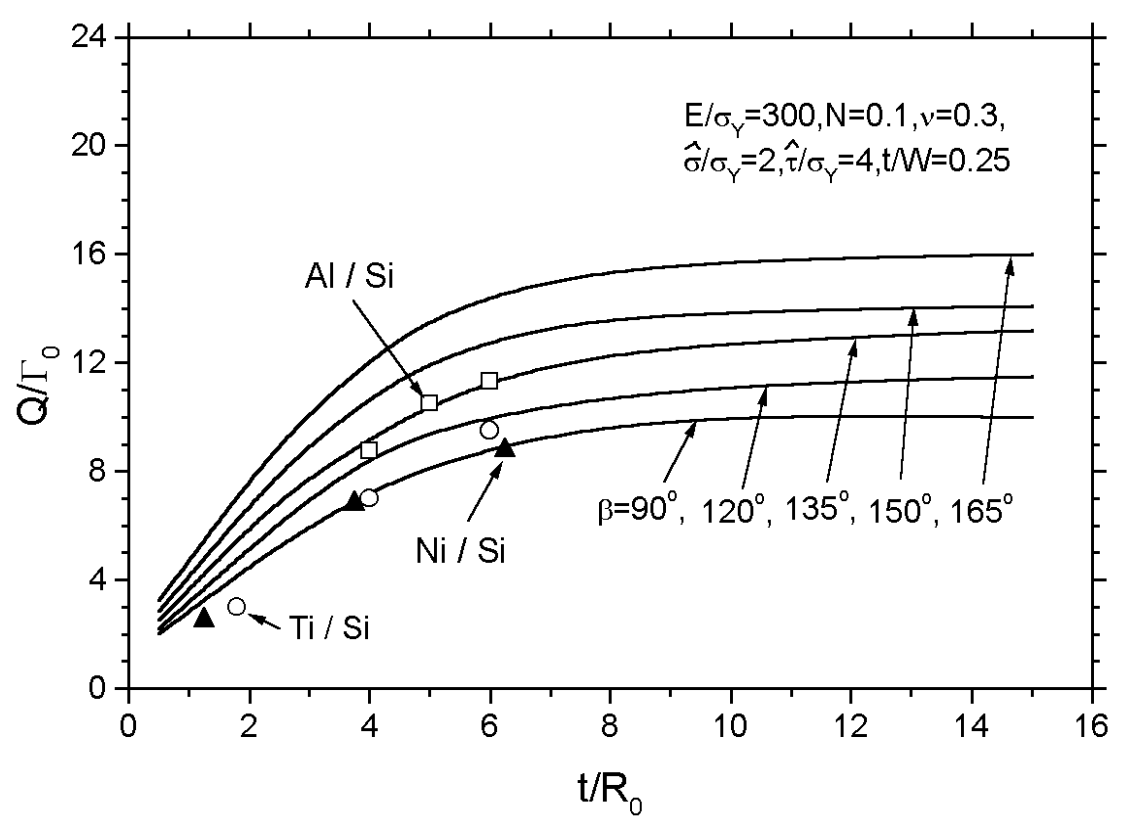

Figure 4. The relations of normalized energy release rate with thin film thickness. 


\section{CONCLUSION}

By the detailed analyses and the scratch experimental research for the $\mathrm{Ni} / \mathrm{Si}$ system in the present research, some important conclusions are obtained as follows: (1) Thin film plastic deformation has the important influence on the advance of delaminated film strap in the scratch test. (2) The interface separation strength and material shear strength have important influence on the failure of thin film/substrate system. (3) The horizontal driving force depends on the thin film or coating layer thickness. With the thin film thickness increase, the horizontal driving force increases and asymptotes to a stable value, which corresponds to the small scale yielding case. (4) In the micro-scratch experiment for the metal thin film on the brittle or ceramic substrate, the film delaminating mechanism shows that as the scratch front approaches the interface, the film is delaminated abruptly.

\section{ACKNOLEDGEMENTS}

The work is supported by National Science Foundations of China through Grants 19891180 and 19925211; and jointly supported by the "Bai Ren" Project from Chinese Academy of Sciences.

\section{REFERENCES}

1. Blees MH, Winkelman GB, Balkenende AR, et al. The effect of friction on scratch adhesion testing: application to a sol-gel coating on polypropylene, Thin Solid Films, 359, 1 (2000).

2. Pistor C, Friedrich K. Scratch and indentation tests on polyoxymethylene (POM), J. Appl. Polymer Science, 66, 1985 (1997).

3. Bull SJ. Failure modes in scratch adhesion testing, Surface \& Coatings Tech., 50, 25 (1991).

4. Maekawa H, Ikeda T, Horibe H, et al. On the scratch pattern of evaporated thin metal film, Quart. J. Japan Welding Society, 12, 262 (1994).

5. Dutta I, Campbell JC. Determination of the adhesive strength of film-substrate interface using the constant depth scratch test, Mater. Res. Soc. Symp. Proc., 390, 49 (1995).

6. Venkataraman S, Kohlstedt DL, Gerberich WW. Continuous microscratch measurements of the practical and true works of adhesion for metal/ceramic systems, J. Mater. Res., 11, 3133 (1996).

7. Jardret V, Zahouani $\mathrm{H}$, Loubet JL, et al. Understanding and quantification of elastic and Plastic deformation during a scratch test, Wear, 218, 8 (1998).

8. Wei Y, Hutchinson JW. Interface strength, work of adhesion and plasticity in the peel test, Int. J. Fracture, 93, 315 (1998).

9. Wei Y, Zhao M, Tang S. Characterization of the fracture work for ductile film undergoing the micro-scratch, Acta Mechanica Sinaca, 18, 494 (2002).

10. Zhao M. Experimental and theoretical researches of failure mechanism of material surface and interface in micro-scratch test, Master thesis of the Institute of Mechanics, Chinese Academy of Sciences, July, 2002. 